\title{
Flight Testing ALHAT Precision Landing Technologies Integrated Onboard the Morpheus Rocket Vehicle
}

\author{
John M. Carson III ${ }^{1,2, *}$, Edward A. Robertson ${ }^{1, \dagger}$, Nikolas Trawny $^{2, \ddagger}$ Farzin Amzajerdian ${ }^{3, \S}$ \\ ${ }^{1}$ NASA Johnson Space Center, ${ }^{2}$ Jet Propulsion Laboratory, California Institute of Technology \\ ${ }^{3}$ NASA Langley Research Center
}

\begin{abstract}
A suite of prototype sensors, software, and avionics developed within the NASA Autonomous precision Landing and Hazard Avoidance Technology (ALHAT) project were terrestrially demonstrated onboard the NASA Morpheus rocket-propelled Vertical Testbed (VTB) in 2014. The sensors included a LIDAR-based Hazard Detection System (HDS), a Navigation Doppler LIDAR (NDL) velocimeter, and a long-range Laser Altimeter (LAlt) that enable autonomous and safe precision landing of robotic or human vehicles on solid solar system bodies under varying terrain lighting conditions. The flight test campaign with the Morpheus vehicle involved a detailed integration and functional verification process, followed by tether testing and six successful free flights, including one night flight. The ALHAT sensor measurements were integrated into a common navigation solution through a specialized ALHAT Navigation filter that was employed in closed-loop flight testing within the Morpheus Guidance, Navigation and Control (GN\&C) subsystem. Flight testing on Morpheus utilized ALHAT for safe landing site identification and ranking, followed by precise surface-relative navigation to the selected landing site. The successful autonomous, closed-loop flight demonstrations of the prototype ALHAT system have laid the foundation for the infusion of safe, precision landing capabilities into future planetary exploration missions.
\end{abstract}

\section{Introduction}

Several near-term planetary science priorities involve robotic landings on the Moon, Venus and comets, as highlighted in the National Research Council (NRC) decadal survey on planetary science. ${ }^{1}$ Future robotic precursor and human missions to Mars will also require landing vehicles. Many compelling science targets are located in terrain that poses risk to safe landing, and future human missions will likely require touch down in the proximity of pre-existing surface assets that pose significant landing hazards. These missions require ALHAT capabilities for onboard autonomy and situational awareness within the GN\&C subsystem to ensure safe and precise soft landing. In fact, the NRC has identified safe and precise landing technologies as a high priority need for future Entry, Descent and Landing (EDL) missions. ${ }^{2}$ These technologies will expand global planetary access for delivering science and exploration payloads directly to targeted surface locations that are currently too risky for mission consideration. Further, direct access to target locations in hazardous terrain regions can minimize the need for long rover traverses, decrease the timeline to initiate science observations, and reduce the time to rendezvous with pre-existing surface assets for robotic sample return or human missions.

The development of technologies for safe and precise soft landing has been ongoing within NASA for more than two decades. The formalized ALHAT project was chartered by NASA headquarters in 2005 with a

*ALHAT Deputy Team Lead, IPA Detailee (to JSC from JPL), AIAA Associate Fellow.

$\dagger$ ALHAT Team Lead.

¥ALHAT Hazard Detection System Lead, AIAA Member.

$\S$ ALHAT Sensors Lead, AIAA Member. 
focus on developing safe and precise landing capabilities for autonomous landing of human or robotic vehicles destined for the Moon. ${ }^{3,4,5}$ These technologies include methods for Terrain Relative Navigation (TRN), longrange altimetry, velocimetry, and Hazard Detection (HD) that are broadly applicable for many solar system destinations. The development of a Hazard Detection System (HDS), Navigation Doppler LIDAR (NDL) for velocimetry and ranging, and a Laser Altimeter (LAlt) for long-range altimetry has primarily been supported through the Human Exploration and Operations (HEO) mission directorate and its predecessors, and the development of TRN capabilities has primarily been supported through the Science Mission Directorate (SMD). Flight testing of the HDS, NDL and LAlt onboard the NASA Morpheus vehicle in 2014 was funded through the HEO Advanced Exploration System (AES) program. The testing was conducted at the Kennedy Space Center (KSC) Shuttle Landing Facility (SLF) where a lunar-like terrain field was constructed based on physical maps and statistical models of rock and crater distributions for smooth lunar maria regions, which are large basaltic terrain regions on the Moon.

The elements of ALHAT provide necessary functions within a GN\&C subsystem to enable precise, safe, and soft landing. Global precision is enabled with TRN, which provides global navigation by matching real-time terrain sensing data with a priori reconnaissance data stored onboard the spacecraft. TRN enables GN\&C to plan and maneuver the lander close to the intended landing site. Note, TRN was not a part of the ALHAT/Morpheus flight testing, but NASA has investigated multiple approaches: passive-optical TRN (e.g., the JPL Lander Vision System $(\mathrm{LVS})^{6}$ ) and active LAlt-based TRN. ${ }^{7,8,9}$ Local precision for soft landing is enabled with direct velocity measurement. The ALHAT NDL uses coherent laser beams to provide ultra high-precision velocity measurements ${ }^{10,11}$ that enable the spacecraft GN\&C system to control lateral and vertical velocities during terminal descent to ensure a soft landing, as well as minimize the growth of navigation position error following the final TRN measurement update. The ALHAT NDL also provides range measurements. Safe landing is enable with HD, which provides local assessment of terrain hazards undetectable in a priori reconnaissance data. The HDS uses a flash LIDAR to create a Digital Elevation Model (DEM) that is analyzed onboard in real time to identify safe landing sites. The HDS also uses the DEM and subsequent LIDAR images to track features and provide navigation position updates and further minimize landing error. While the LIDAR-based HD functions in both illuminated or shadowed/dark conditions, ${ }^{12,13}$ there also exist passive optical techniques ${ }^{14}$ for well-illuminated conditions.

This paper provides an overview of the 2014 ALHAT/Morpheus flight test campaigns and highlights the performance and lessons learned with the ALHAT prototype HDS, NDL and LAlt. Section II gives an overview of these three ALHAT prototype sensors, along with their locations integrated onto Morpheus for the flight testing. A description of the six flight tests, including the trajectory profile and some details on each of the campaigns, follows in Section III. Section IV provides an assessment of the performance for each of the ALHAT components throughout the flight campaigns. Some comments on next steps toward infusion into spaceflight missions are included in Section V, and closing remarks are provided in Section VI.

\section{ALHAT Sensors and Vehicle Integration}

The ALHAT sensors shown in Figure 1 include a three-beam NDL ${ }^{10}$ that provides precise velocity measurements, a long-range LAlt, ${ }^{9}$ and a flash LIDAR-based ${ }^{15} \mathrm{HDS}^{16,12}$ that provides real-time Hazard Detection (HD) ${ }^{17}$ for onboard determination of safe landing sites.

The prototype LAlt uses a moderate power laser operating with an eye-safe energy level at $1.57 \mu \mathrm{m}$ wavelength for terrestrial operation and testing. The laser generates pulses a few nanoseconds long that allows for high-precision distance measurements when a high resolution Time-of-Flight (TOF) counter is employed. The prototype LAlt in Figure 1 (left) has significantly longer range and precision than required for the Morpheus flight tests where it was used as a standard altimeter; the original design intent for the LAlt was long-range TRN from $15+\mathrm{km}$ altitude. Altitude measurements with $0.5 \mathrm{~m}$ precision from about $20 \mathrm{~km}$ are more than sufficient for mitigating the drift within the Inertial Measurement Unit (IMU) and provides an accurate vehicle position estimate. The measurements of the terrain contours required for Terrain Relative Navigation requires similar operational range and precision specifications. The current sensor significantly exceeds these performance requirements as it can operate from over $50 \mathrm{~km}$ with about $5 \mathrm{~cm}$ precision. Some 

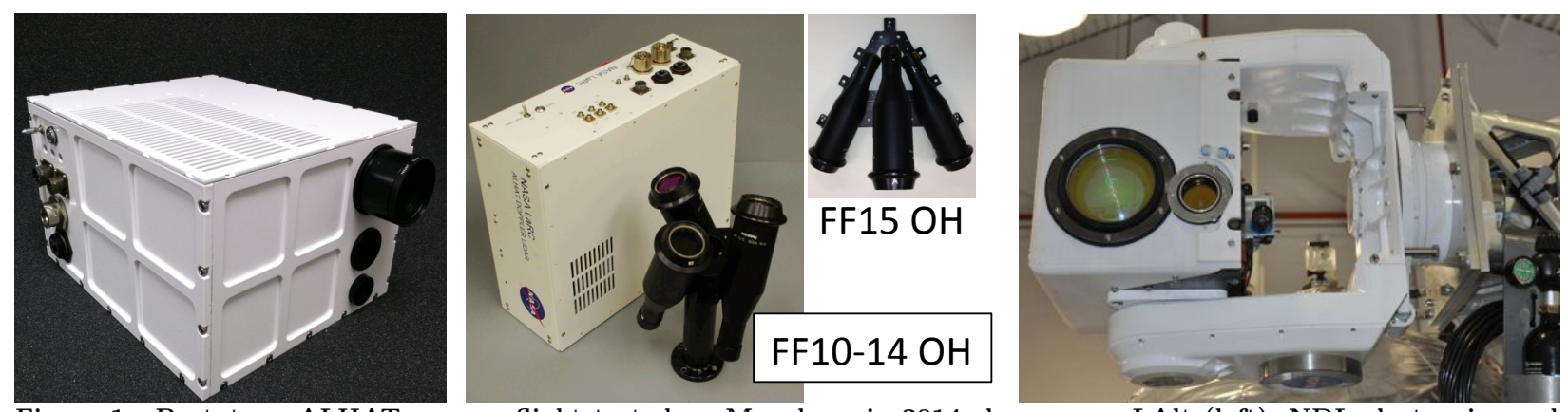

Figure 1. Prototype ALHAT sensors flight tested on Morpheus in 2014: long-range LAlt (left), NDL electronics and two versions of optical head (center), and HDS gimbaled flash LIDAR (right).

features of the LAlt include multi-return and automatic detection threshold capabilities: the sensor generates three range measurements for each transmitted pulse to facilitate operation in the presence of clouds, and $\underline{\text { the }}$ automatic detection threshold function enables reliable operation with a low false alarm rate over its operational range. ${ }^{9}$

The NDL provides accurate vector velocity and altitude data during the descent phase from a few kilometers above the surface. ${ }^{10,11}$ The NDL data can be used by a GN\&C system to minimize Navigation knowledge error growth in velocity and position estimates and to tightly control vertical and lateral velocities during terminal descent to ensure a soft touchdown. The NDL uses a Frequency Modulated Continuous Wave (FMCW) technique and optical homodyne detection to obtain both range and velocity data. A relatively low power, single frequency laser operating at an eye safe wavelength of $1.55 \mu \mathrm{m}$ is used as the master oscillator. The frequency of this laser is modulated linearly with time. A portion of the laser power is amplified to be transmitted and the rest is used as the local oscillator (LO) for optical homodyne detection. The LIDAR transmits three laser beams into three fixed directions relative to each other, and the return signals are directed to three corresponding photo-receivers. The digitized outputs of the three photo-receivers are processed by a Fast Fourier Transform (FFT) algorithm to extract line-of-sight (LOS) velocity and range data. The platform vector velocity is determined from the LOS velocities measured along the three laser beams by using a priori pointing knowledge. Using all three simultaneous LOS range measurements allows determination of the vehicle altitude relative to the local ground without the need for vehicle attitude angle data from a separate sensor. The use of three beams to compute altitude also reduces the effect of terrain features such as boulders and craters when compared to a single beam radar or LIDAR altimeter. The prototype NDL that was flight demonstrated on Morpheus is capable of measuring vehicle velocity and altitude with $0.2 \mathrm{~cm} / \mathrm{s}$ and $30 \mathrm{~cm}$ precision, respectively, at a maximum velocity and altitude of $70 \mathrm{~m} / \mathrm{s}$ and $2.2 \mathrm{~km}$, respectively.

The HDS consists of a flash LIDAR mounted on a two-axis gimbal, a Northrop Grumman LN200 inertial measurement unit (IMU), a dedicated compute element (CE), and a power distribution unit (PDU) and battery box. The HDS CE combines a Xilinx FPGA, a multicore Tilera Tile-64 multicore processor, and a COTS PC/104+ x86 single board computer, providing precise time stamping and time synchronization, high-performance computing, and onboard data logging. The 3-D imaging flash LIDAR built by LaRC is a second generation, compact, real-time, air-cooled, $20 \mathrm{~Hz}$ time-of-flight sensor system based on 3-D imaging camera technology developed by Advanced Scientific Concepts (ASC). ${ }^{18}$ The LaRC LIDAR integrates a $1.064 \mu \mathrm{m}$ class IV laser, $1^{\circ}$ field of view (FOV) f/7.3 receiver optics, $1^{\circ}$ divergence transmitter optics, and a 128 pixel $\times 128$ pixel focal plane array (FPA) which resides in the ASC camera. The flash LIDAR achieves a range precision of $8 \mathrm{~cm}(1-\sigma)$, and an absolute range accuracy of better than $35 \mathrm{~cm}$ over the full operational range of $50 \mathrm{~m}$ to over $750 \mathrm{~m}$. Using the gimbal, the flash LIDAR was scanned across a $60 \times 60 \mathrm{~m}$ area of the hazard field to form a mosaic of 117-120 individual range images, with a nominal 50\% crosstrack and $20 \%$ downtrack overlap fraction, which HDS combined onboard to create a seamless, $10 \mathrm{~cm} /$ pixel digital elevation map (DEM). This DEM was then evaluated for slope and roughness hazards at lander scale ${ }^{17}$ and combined into a safety probability map, from which the five safest landing sites are chosen and communicated to the 
vehicle GN\&C system. To this end, several intermediate products are computed:

Leg-pad map The leg-pad map stores the position in space of the lander's footpads on the DEM. We(?) assume some conformity of the pad with the surface and use the median DEM elevation of a $3 \times 3$ pixel area for the position of the $25 \mathrm{~cm}$ diameter Morpheus footpad.

Effective slope map The leg-pad map is queried to compute the slope of a lander of a certain diameter (3.4 $\mathrm{m}$ for Morpheus) positioned at a given location, and in various orientations. For all possible placements of the lander pads (the fourth is either on the surface or elevated) the worst slope is taken for each pixel every $1 \mathrm{~m}$. For expediency only the placements deviating $10^{\circ}$ from the lander heading were considered rather than the full $45^{\circ}$ span.

Effective full roughness map Roughness hazardness is estimated probabilistically. By examining at each pixel and all the surrounding pixels under the lander (full roughness) the largest deviation from the lander bottom to all possible slope planes for that pixel, the chance that there is a roughness hazard under the lander is assessed in conjunction with the associated elevation uncertainties of those pixels. As with slope hazard assessment, the estimates are computed $1 \mathrm{~m}$ apart but based on the full resolution DEM data.

Hazard probability map Roughness probability maps and slope maps are combined into a hazard probability map. To take into account navigation uncertainty $(1 \mathrm{~m}, 1-\sigma)$ the hazard probability map is convolved with a Gaussian kernel with space constant $1 \mathrm{~m}$ to produce the final safety probability map.

The safe site selection algorithm applies a morphological dome operator to detect all the regional maxima in the surface represented by the safety map. The peaks in the regional maxima are true unambiguous peaks, i.e., all the pixel neighbors have values smaller than the peak value. To prevent multiple peaks from clustering in nearby locations, the algorithm enforces a parameterized minimum separation among the peak locations. The process assembles a probability-ranked list of peaks and reports the top five peak locations to the lander.

Following hazard detection, the HDS selects and tracks a an easily identifiable feature, i.e., a large rock in the DEM, to provide hazard relative navigation (HRN) updates to the vehicle GN\&C system. The difference between expected and observed image pixel location of the feature can be used by the vehicle navigation filter to correct and limit position error growth relative to the DEM. In flight, each incoming LIDAR image is converted to a single flash DEM. A template centered on the dominant terrain feature in this DEM is first flattened and then correlated with the HD DEM in a search region around the predicted location. The location of the best match is computed with subpixel accuracy using biquadratic interpolation of the correlation surface. Each match is then evaluated against quality metrics on the correlation scores, the measurement covariance, and the correlation peak quality (sharpness) before being passed to the host vehicle. The HRN measurements the host vehicle receives include the map location of the surface feature being tracked, the location of the surface feature relative to the HDS, in a sensor-fixed reference frame, and the uncertainty of the HDS-relative feature position. Note that in flight the decreasing slant range to the landing site results in an increasing mismatch between map and image resolution, or, equivalently, a smaller and smaller LIDAR footprint in the map. This, together with the limited depth of field of the LIDAR eventually precludes successful HRN measurements as the vehicle approaches the landing site.

Enabling the ALHAT precision landing capabilities requires detailed calibration of the individual ALHAT sensors, as well as precise alignment knowledge of the sensors integrated onto the host vehicle. Each of the ALHAT sensors underwent individual alignment and calibration testing to characterize measurement accuracy and precision, as well as stand-alone ground and flight tests prior to Morpheus to evolve the sensor designs and mature the algorithms. Details of prior ALHAT-specific flight and development tests can be found within numerous ALHAT publications. ${ }^{11,13,16,19-22}$ Demonstration of the prototype ALHAT sensors onboard Morpheus involved a detailed preparation, integration and verification campaign to metrology align the ALHAT sensors and validate their measurements with the Navigation filter prior to tether tests and free flight tests. ${ }^{23,24}$ 

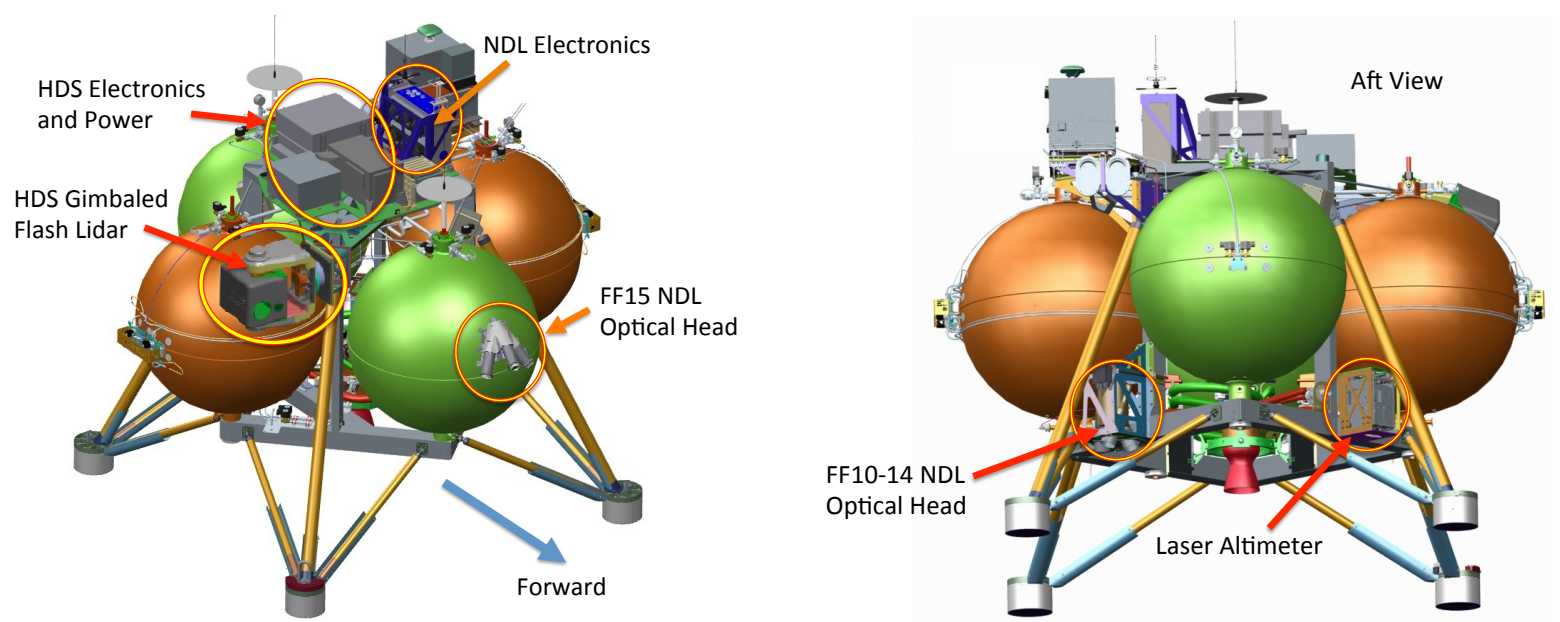

Figure 2. Illustrations of ALHAT sensors and electronics onboard the Morpheus $1.5 \mathrm{~B}$ vehicle.

\section{Test Objectives and Flight Profiles}

The Morpheus/ALHAT free reference flight profile, shown in Figure 3, was designed to test the ALHAT safe, precision landing functions and sensors during the approach and terminal descent phases of the simulated lunar landing trajectory at the KSC SLF. Three open-loop free flights (FF10, 11, \& 12) were conducted using the Morpheus terrestrial navigation system (GPS + IMU + COTS altimeter), called VTB Nav, to evaluate the performance of the ALHAT LAlt, NDL, HDS and the associated extended Kalman navigation filter, called ALHAT Nav. In addition to verifying ALHAT system performance during dynamic flight, the openloop free flights provided confidence in the ability of the vehicle guidance system to transition from ALHAT navigation to Morpheus navigation in the event that the ALHAT solution diverged from the defined flight corridor. The three open-loop Morpheus/ALHAT flights were followed by three closed-loop flights using the ALHAT navigation solution. Two of the closed-loop free flights were performed in May 2014, and the final flight (FF15) was completed in December 2014. See Table on the left of Figure 4 for a summary of the planned flight test campaigns, along with the resultant flight trajectories overlaid on an SLF terrain image (Figure 4 (right)).

As illustrated in Figure 3, the free flight profile consisted of a vertical ascent phase to an altitude of approximately 245 meters, transitioning via a pitch maneuver into the approach phase at a slant range of approximately 450 meters from the initial target. The ALHAT HDS was designed to operate from a slant range of up to $1000 \mathrm{~m}$, which would require a flight trajectory with roughly twice the altitude and down track distance achieved during the FY2014 Morpheus/ALHAT free flights.

Shortly after pitch-over, the vehicle dynamics were sufficiently damped to initiate terrain imaging and Digital Elevation Model (DEM) generation in support of autonomous Hazard Detection and Avoidance (HDA). The 60x60 meter DEM was centered on the initial landing target and was constructed from 117 to 120 flash LIDAR range images over a period of approximately 6 seconds. The HDS subsequently analyzed the DEM and delivered a prioritized list of safe landing targets to the Morpheus Autonomous Flight Manager (AFM) software to support the GN\&C divert maneuver. The entire HDS process, from the start of terrain mapping to the delivery of the safe site list, required less than thirteen seconds. If the top-ranked safe site identified by the ALHAT HDS was within two meters of the center of the landing pad, the AFM selected that site as the new landing target and the GN\&C system initiated the divert maneuver. Otherwise, the GN\&C automatically diverted to the center of the pad following a 14.1 second timing trigger.

The Morpheus vehicle was designed for lunar missions, not as a terrestrial test bed. As a result, the terrestrial flight profile was constrained by the maximum thrust of the main engine, which limited the flight duration to approximately 100 seconds (TBR) plus a modest safety margin. The Morpheus/ALHAT trajectory was also constrained by the ability of the roll control thrusters to counteract aerodynamic torques, 


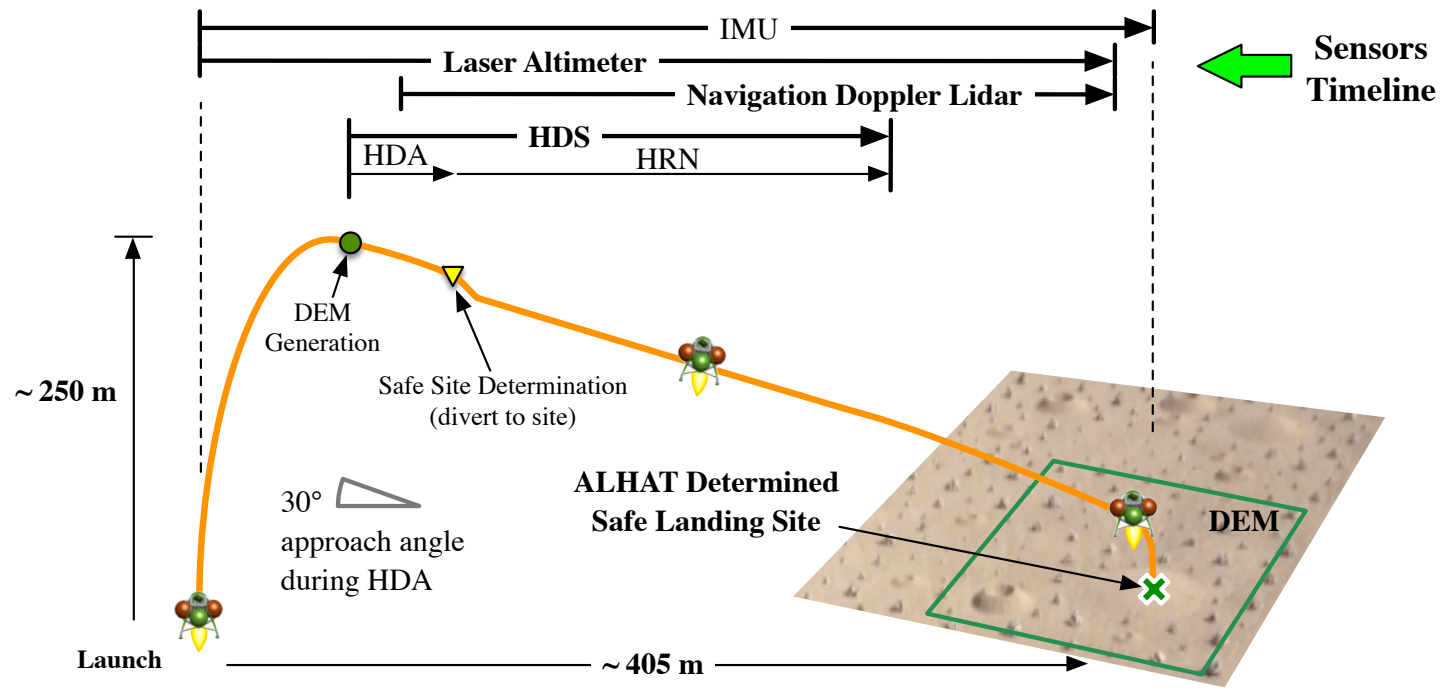

Figure 3. Flight profile and ALHAT timeline during open-loop and closed-loop Morpheus flight tests.

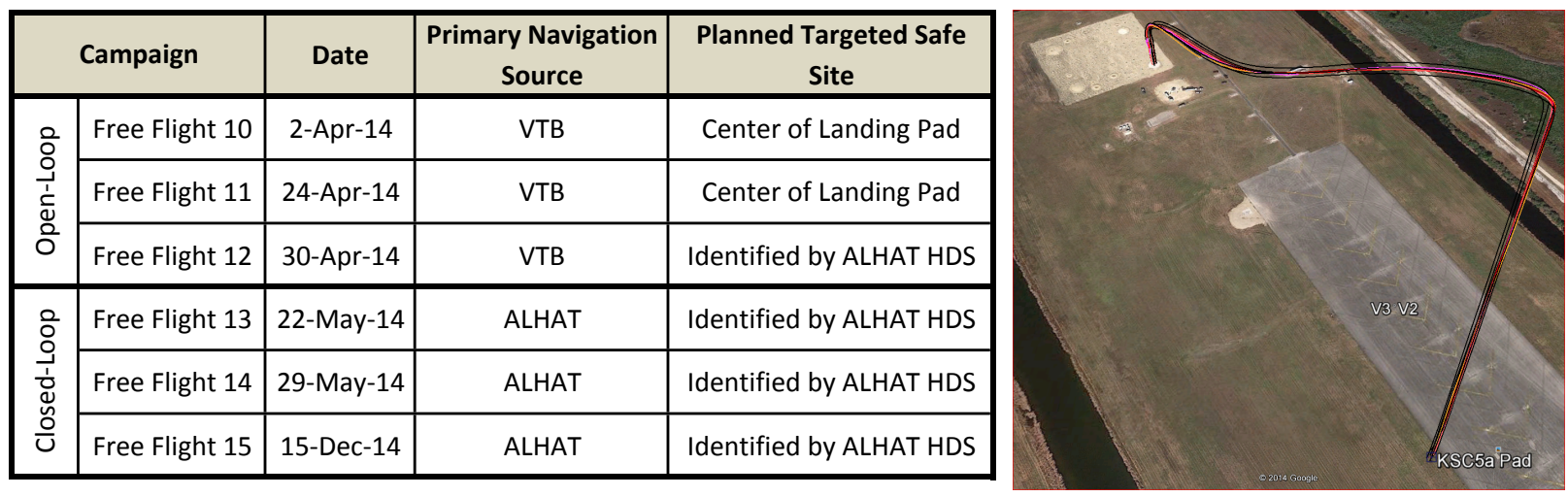

Figure 4. Morpheus trajectory profiles during ALHAT flight testing.

which limited the maximum airspeed to roughly $35 \mathrm{~m} / \mathrm{s}$ (TBR). In combination with the motion of the Morpheus lander, the local winds also affected the distribution of the mass of hot, turbulent air induced by the main engine exhaust plume. This plume resulted in intermittent signal loss for the downward pointing LAlt and NDL sensors, and was also observed to adversely affect the quality of the flash lidar images when the wind was blowing towards the hazard field. As a result, launch constraints were imposed on wind magnitude and direction and the NDL optical head was relocated to the forward tank for FF15 to reduce lidar-plume interaction.

The ALHAT system for safe, precision landing utilizes TRN during the powered descent trajectory to reduce position errors relative to a map-based target - potentially by an order of magnitude or better. For missions utilizing onboard hazard detection, TRN also accurately positions the lander relative to the vetted landing site area prior to the transition to the approach phase. A TRN subsystem was not included in the Morpheus/ALHAT flights primarily because the Morpheus GN\&C executes the ALHAT and vehicle navigation filters in parallel and, then, compares the two navigation state estimates to ensure that the lander remains within a tightly constrained flight corridor. As a result, it was necessary to synchronize the two navigation states just prior to liftoff. In addition, the benefit/cost trade of incorporating TRN was judged to be unfavorable because of the short duration and low maximum altitude of the flight profile, the presence 
of man-made structures and vegetated terrain, and the fact that multiple TRN approaches were successfully tested on a helicopter over lunar and Mars analog terrain during ALHAT Field Test 3 in 2009.

\section{ALHAT Flight Test Performance}

The two primary objectives for the Morpheus/ALHAT flight campaigns were to demonstrate the precision landing capability of the ALHAT navigation filter and sensors and to land in close proximity to a valid safe landing site identified by the ALHAT HDS. Ideally, both of these objectives would have been accomplished during every closed-loop flight. Ultimately, however, these objectives were demonstrated separately in different flights.

The first three free campaigns were open-loop exploratory flights used to evaluate ALHAT navigation results against the VTB GPS-based trajectories and to test the operation of the HDS. During all three open-loop flights, the HDS identified valid safe landing sites and, during Free Flight 12, the safe landing site list developed by the HDS was successfully utilized by the Morpheus GN\&C as the updated landing target. The open-loop flights provided sufficient confidence to proceed with closed-loop testing, but also identified concerns with LAlt and NDL availability due to plume-induced signal degradation, navigation response to HRN measurements, and with the modest and variable map-tie error between the HDS DEM and the surveyed hazard field. During Free Flights 13 \& 14, the Morpheus landed lifted off and completed much of the trajectory using ALHAT navigation. However, shortly after the HDS DEM generation and safe site identification were completed, the ALHAT navigation solution exceeded the boundaries of the allowable flight corridor and the vehicle switched to VTB navigation. These flight corridor violations occurred due to the relative scarcity of three-beam NDL measurements combined with the transient response of the ALHAT navigation filter to the processing of HRN measurements. The HDS identified valid safe landing sites during both FF 13 \& 14. During FF13 the top-ranked site did not fall within two meters of the surveyed center of the landing pad. During FF14, however, the lander accepted the HDS target and completed the landing trajectory on VTB navigation, thus satisfying one of the primary test objectives.

Four key changes were implemented in preparation for the final closed-loop Morpheus/ALHAT free flight campaign. First, the NDL optical head was relocated from the bottom of the vehicle to the forward tank to reduce the plume-induced effects and increase the availability of three-beam NDL data. Second, a liftoff wind placard was established to avoid tailwinds that could push hot, turbulent air in front of the NDL and HDS sensors during the approach phase. Third, the altitude at which the processing of LAlt and NDL measurements halted and the vehicle switched to dead reckoning was increased to 30 meters. This prevented the processing of noisy measurements from the dust cloud kicked up by the lander main engine during terminal descent. Fourth, the weighting of the HRN measurements in the ALHAT navigation filter was adjusted to reduce the magnitude of its response. As a result of these configuration changes, FF15 was performed using only the ALHAT navigation solution, from liftoff through touchdown, thus satisfying the second primary test objective. The HDS once again provided valid safe sites during FF15, but the top-ranked safe site was not on the landing pad.

The three ALHAT sensors work together through ALHAT Nav to provide terrain-relative state measurements that enable autonomous and safe precision landing. The open-loop campaigns provided flight test data for revising the ALHAT Nav filter without risking the vehicle to closing the loop on the new Navigation state. The switch-over to VTB Nav in FF13 and FF14 was due primarily to unexpected affects of the terrestrial test environment on the NDL, which will be discussed later, but there were also some unresolved interactions between ALHAT Nav with HRN measurements. The open-loop tests were incapable of providing sufficient data to debug ALHAT Nav and HRN interactions. The GN\&C software architecture for Morpheus fed the HDS the primary Navigation state, which during open-loop testing was the VTB Nav state. The HDS tracks features during HRN based on the DEM constructed during HD and based on the Nav solution that is provided. Since the VTB Nav state was close to truth, the open-loop flights did not provide sufficient test conditions to iron out all interactions between ALHAT Nav and HDS during HRN. The FF13 and FF14 closed-loop data was used for further HRN revisions that fed into the successful closed-loop flight demonstration of ALHAT Nav during FF15. The plots of Figure 5 show how close the ALHAT Nav and 
VTB Nav position and velocity solutions were during the final closed-loop flight. Since the VTB Nav is not an independent truth source, the two Nav solutions are not expected to perfectly overlay. There is inherent time lag in the VTB Nav measurements, and the the DEM used for HRN has map-tie error due both the ALHAT Nav errors, as well as sensor alignment errors.
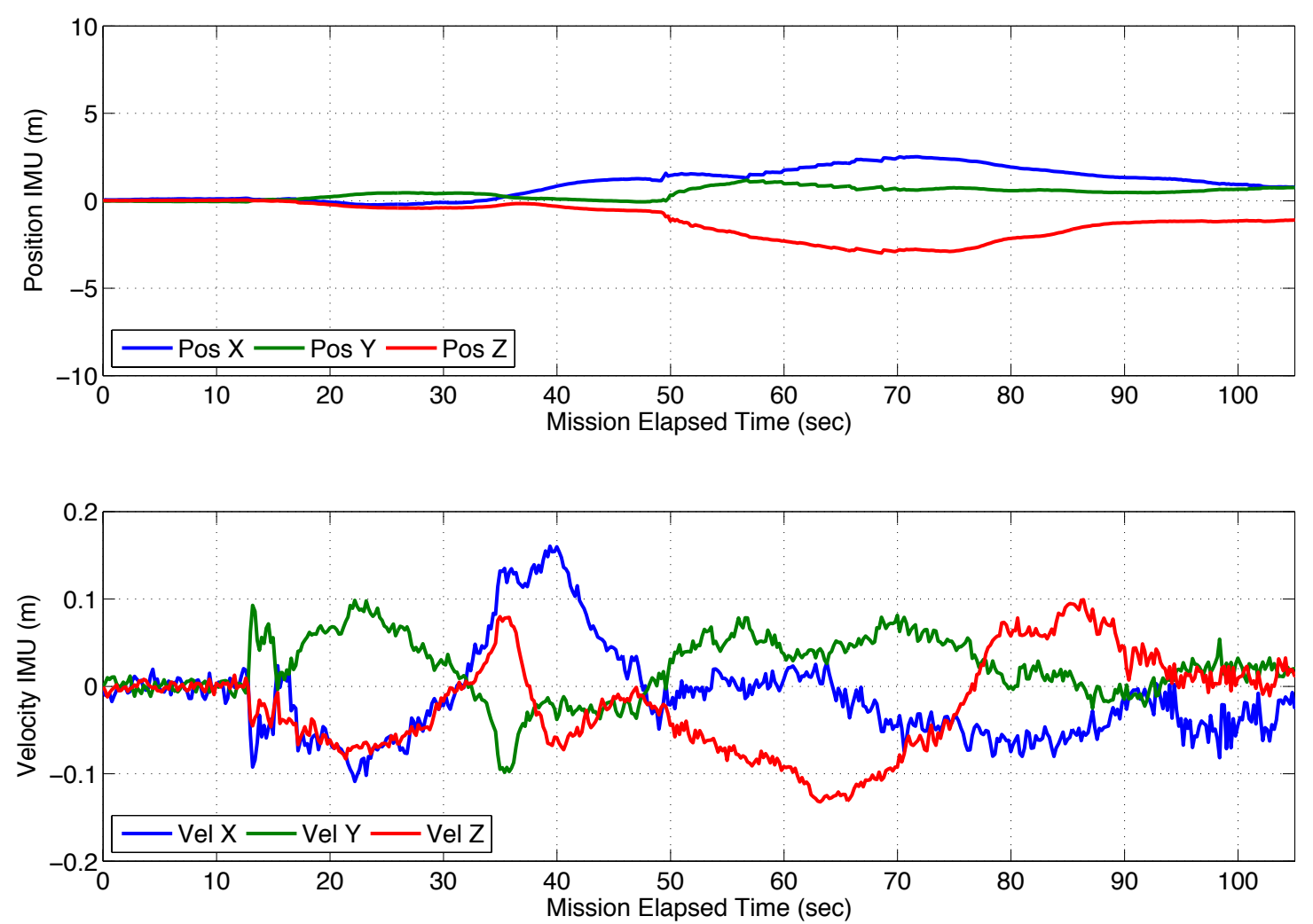

Figure 5. The difference between ALHAT Nav and VTB GPS-based Nav was minimal during Free Flight 15.

The HDS algorithms were designed to select the top five sites ranked by safety probability in an onboard analysis of the DEM. Cite HD algorithm paper (Toni, Andres and I). The algorithm performed flawlessly in all truck, helicopter and Morpheus flight tests. Algorithm was the result of multiple years of R\&D to develop the right approach to HD, which was through probabilistic assessment and ranking of safety. The algorithm considers not only the lander hazard tolerances, but also the precision of the LIDAR sensor and the uncertainty in the Navigation state when determining the safest landing sites. There is no a priori consideration for the terrain, such as locations of landing pads. The ALHAT system knew nothing about the landing pads or safest locations for landing prior to each flight onboard Morpheus, and the ALHATdetermined safe landing sites were solely a function of the DEM generated in real time during each flight from onboard acquired LIDAR images. The ALHAT system provided Morpheus GN\&C with the top list of sites, and Morpheus GN\&C then determined if the HDS site would be utilized or a pre-existing survey location. A threshold was put in place for terrestrial test considerations such as ease of vehicle recovery by the crane following each flight. As such, the threshold required the Morpheus GN\&C to only land on the pad, so when the safest HDS site was outside of the threshold, then the pre-determined pad location was used.

In flight, the HDS showed very repeatable timing performance. The mosaic scan completed in 5.8-6.0 s. The incoming range images were processed in parallel, and the DEMS were generated in 9.4-10.7 s, with an additional 0.6-0.8 s for DEM finalization. The actual hazard detection and safe site selection process executed in 1.0-1.3 s. The total process from triggering the mosaic to reporting the safe sites to the host vehicle took 11.3-12.9 s, which was well within the 11.2-14.1 s prediction window, established through detailed 
Monte Carlo simulations. ${ }^{25}$ All DEMs achieved greater than $99.6 \%$ data density, with small holes due to shadows from rocks and craters, and the flat, gravel-covered landing pad was reconstructed with an elevation standard deviation of 4-6 cm (1- $\sigma)$. The map tie error was 0.9-3.2 m mostly in downtrack direction All safe sites reported by the HDS in flight were truly safe as evaluated against the ground truth safety map, and concrete landin pad was always within the top 5 reported sites, meeting the test objectives. Following issues with software bugs prior to FF14, dust clouds obscuring large parts of the lidar images, or with the feature moving outside of the LIDAR field of view due to navigation errors, ${ }^{26}$ the ALHAT navigation filter processed 7 HRN measurements during Freeflight 15, with the feature staying within the LIDAR field of view almost throughout the entire descent. Post-processing confirmed that that the rock was tracked accurately, thus demonstrating full functionality of HRN.

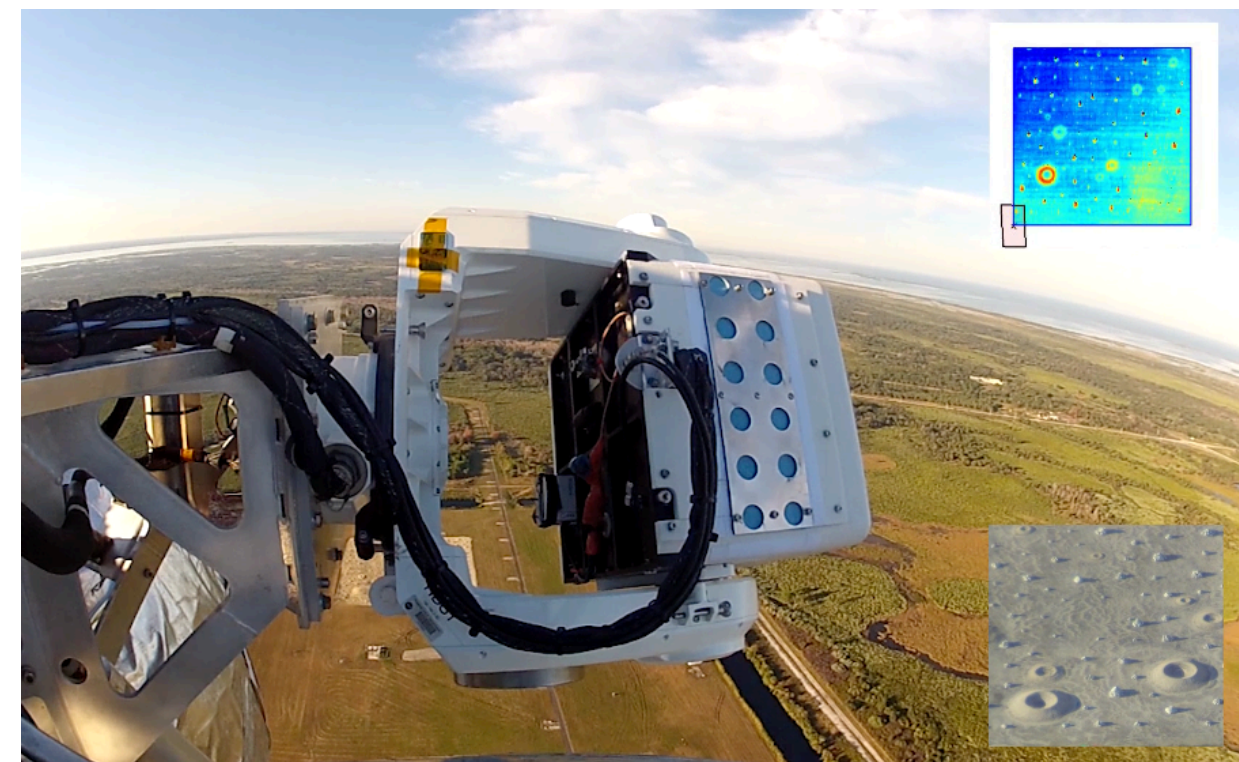

Figure 6. HDS performs in-flight mosaic imaging of terrain to construct DEM (upper right) and analyze for safe landing sites. Co-boresighted witness camera images of terrain being scanned shown in lower right.

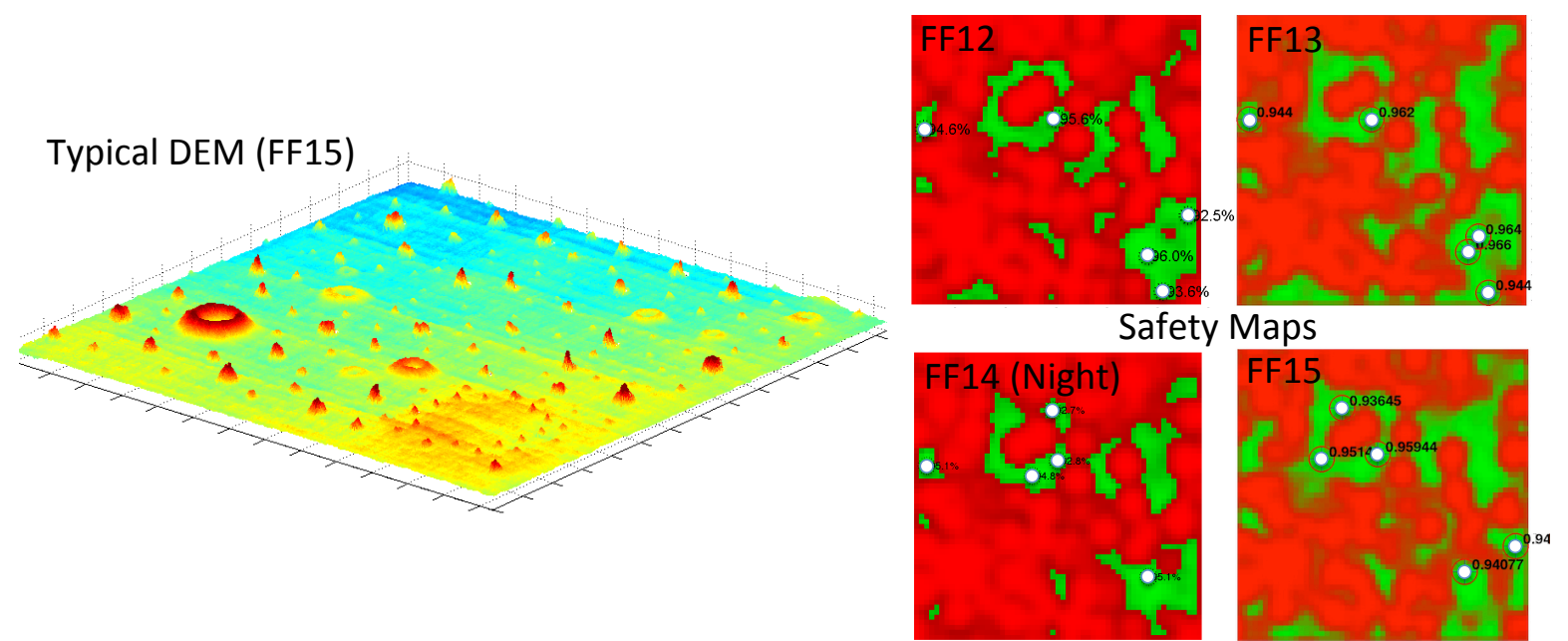

Figure 7. All HDS-determined sites were always safe in truth during the ground, helicopter and Morpheus testing. Typical DEM (at left) and safety maps (at right) with top 5 safe sites (white dots within green safety regions) generated onboard and in real time during Morpheus flights.

The NDL provided critical velocity data for navigating and landing at the designated location defined 
by the HDS. Figure 8(left) shows the NDL velocity measurements during the last flight (FF15). For this flight, the NDL optical head was installed on one of the Morpheus fuel tanks pointing toward the direction of flight as shown in Figure 2. The red curves show the NDL LOS velocity for each of the three beams over the duration of the flight. The blue and green curves are the velocity estimates, projected along the LIDAR beams, from the vehicle navigation system (using GPS and IMU data) and ALHAT navigation system (using NDL and IMU) showing excellent agreement between them. The resolution of LOS velocity measurement is $2 \mathrm{~mm} / \mathrm{sec}$ limited by the FFT processing algorithm. The NDL velocity precision, defined as random noise about its mean, was measured to be $1.7 \mathrm{~cm} / \mathrm{sec} 1$-sigma, dominated by the vehicle vibration. The accuracy of velocity was estimated analytically to be well under $1 \mathrm{~cm} / \mathrm{sec}$ without including the bias error due to errors in the registration of the laser beams pointing angles. The NDL velocity precision and accuracy demonstrated in the Morpheus flights are about an order of magnitude better than those required for precision and/or well-controlled soft landings. Figure 8(right) provides the NDL altitude data for the same flight, showing the three LOS measurements of range to ground. One of the beams looking along the flight path measuring a longer distance while the other two beams looking to the sides but still tilted forward have their measured plots almost overlap. From these three LOS range, the vehicle altitude relative to local ground is calculated in real-time without attitude data from the IMU. ${ }^{11}$ Although the NDL altitude data was not utilized by the ALHAT navigation filter, the post flight analysis showed its usefulness in reducing the vehicle position estimation.
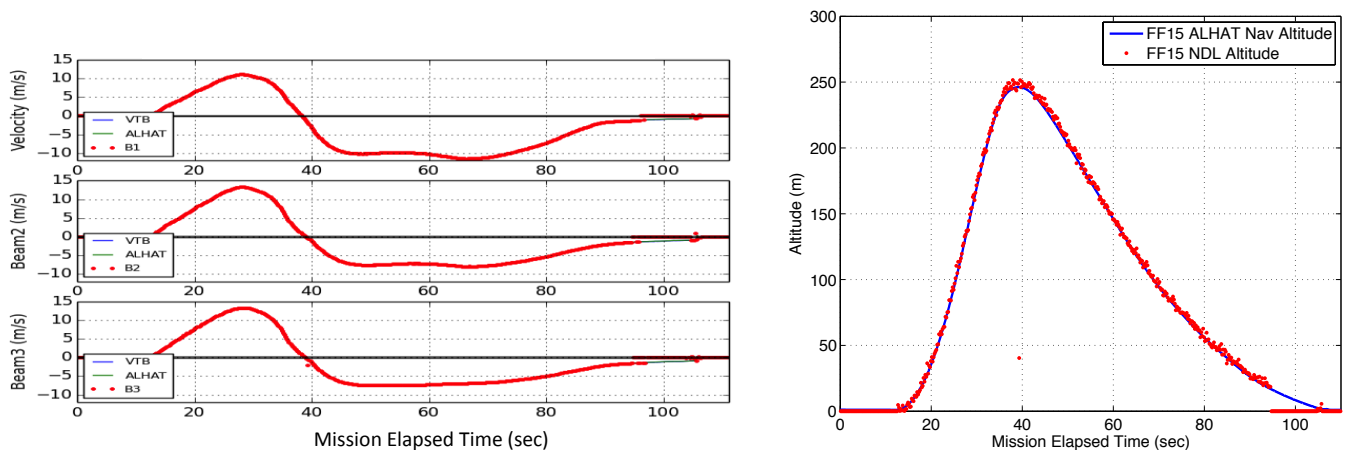

Figure 8. LOS velocity (left) and range (right) measured by each of NDL beams during FF15. NDL velocities (red) is compared with the velocities estimated by the vehicle and ALHAT navigation systems are shown (blue and green). The LOS range data from each beam (2 of them overlap) and resultant altitude data also agreed very well with data from other sensors.

The plots of Figure 8 show some data dropouts at beginning of the flight and the last few seconds of the flight due to the dense dust cloud created by the engine during on takeoff and landing (Figure 9). The loss of LIDAR data in dust plume was expected and the ALHAT landing system was designed to dead-reckon to a soft landing by utilizing the precision vector vertical provided by the NDL prior to the creation of dust plume during the final stage of descend.

During the first five flights (FF10-14), there were considerable NDL data dropouts, especially Beam B, when the NDL optical head was installed on the aft, lower deck of Morpheus approximately $1 \mathrm{~m}$ from the main engine, as illustrated in Figure 2. The data dropouts were due to a turbulent air mass generated by the extreme engine heat, which affects the local air up to the vehicle midline, as seen in the thermal image of Figure 10 (left). The FF14 NDL data in 10(upper right) is representative of FF10-14 data dropouts; here, Beam B is significantly affects, and several segments of Beam C are also missing. The turbulent air degrades the signal coherence thus affecting the heterodyne detection efficiency. The signal dropout pattern varied for FF10-14 due to the wind direction pushing the heated air into the path of one beam or another. This was the reason for re-locating the NDL optical head in FF15 to the above the midline, away from the engine, and forward canted to avoid the engine heat plume. The relocation eliminated the data dropouts, as seen in 10(lower right).

Although the LAlt was designed for high altitude TRN, the sensor provided good range data during the Morpheus flights when used as a short-range sensor. Figure 11 shows an example of the LAlt data 

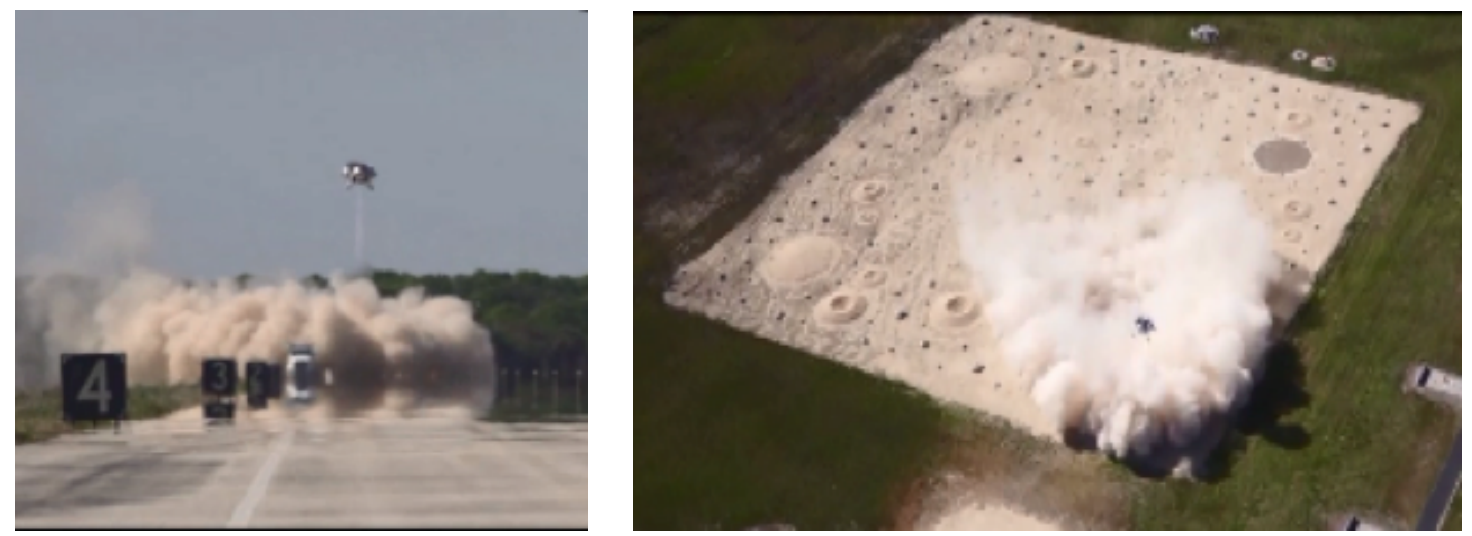

Figure 9. Dust clouds created by the Morpheus engine during takeoff (left) and Landing (right).
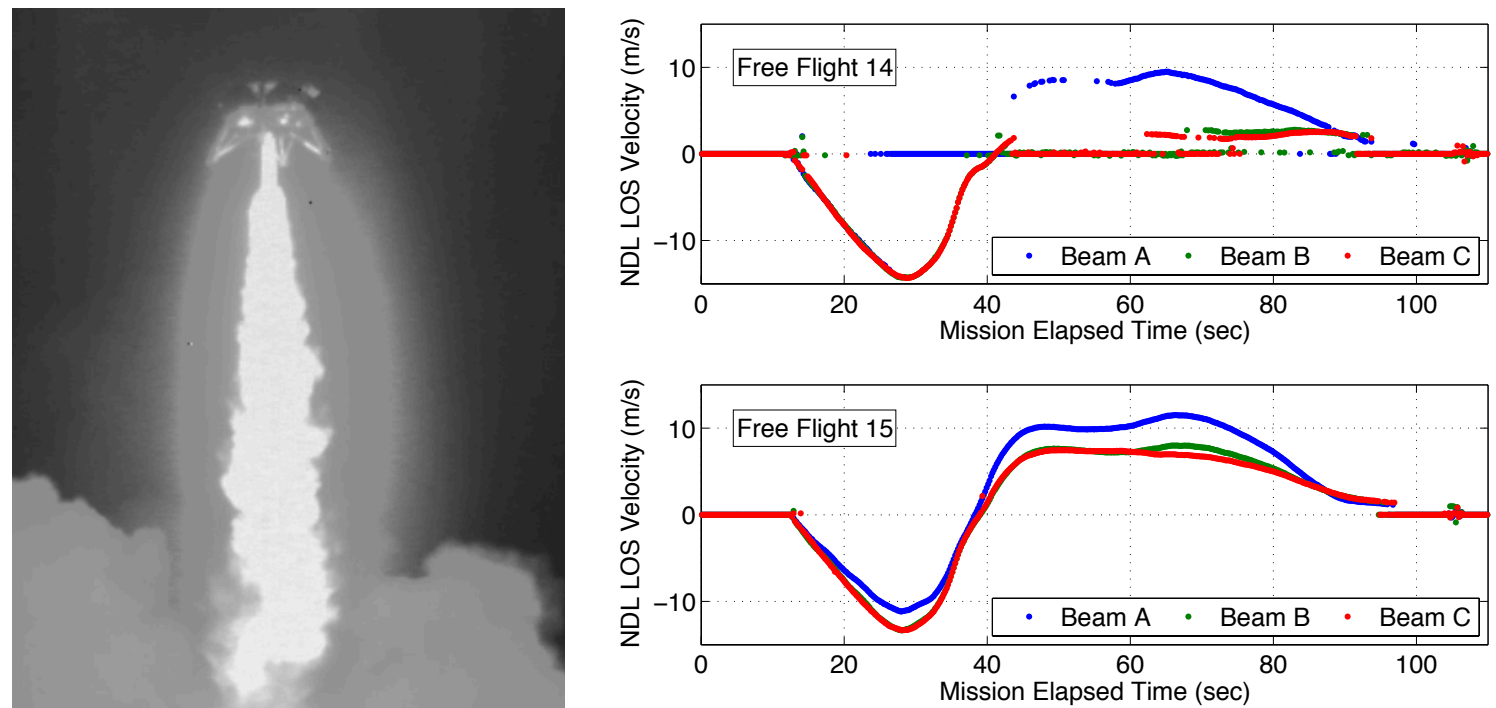

Figure 10. Thermal heating of air ( IR image at left) from the Morpheus engine plume degraded NDL measurements in FF10-FF14 due to the original, lower-aft positioning of the sensor (FF14 data at right, top). Relocating the NDL for FF15 to a forward, above-midline position enabled successful NDL measurements throughout the flight (right, bottom).

compared to the Nav altitude estimate from the Morpheus GN\&C system. The difference between the LAlt measurement and the altitude estimate is due to the Morpheus vehicle pitching over at peak altitude, which points the LAlt off nadir. The plot data is not corrected here for that pitch offset. the LAlt range is altitude measurements is of the order of $1 \mathrm{~m}$ some of which is due to the terrain variations. Like NDL, the laser altimeter is also affected by the dust cloud on landing. 


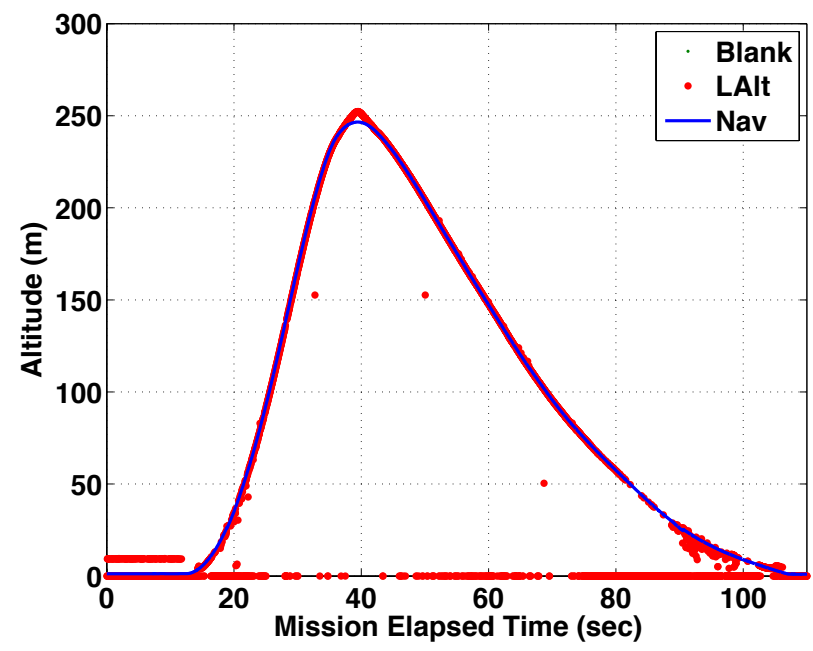

Figure 11. Laser Altimeter measurement compared with NDL and vehicle altitude data.

\section{Steps Toward Spaceflight Infusion}

The flight demonstration of the prototype ALHAT sensors onboard Morpheus has been an important milestone toward validating the capabilities for maturation and implementation within the GN\&C subsystem of a space lander mission. The ALHAT team has made use of the terrestrial flight test data and experience to further mature the sensor designs toward flight. Efforts are underway to implement Engineering Development Units (EDUs) for the HDS and the NDL that are ready for infusion into near-term robotic science missions, including Discovery and New Frontiers missions, and well as near-term Mars missions after Mars 2020.

The HDS prototype has reliably demonstrated the capability to generate onboard maps, detect hazards, and determine safe landing sites all in real time. The prototype design and operations concept was developed for a large human-scale lander requiring large maps and long range imaging. As a result, the prototype system is composed of heavy, mid-TRL components including the long-range gimbaled LIDAR, terrestrial multi-core processor and a computationally-complex HD algorithm. The Size, Weight and Power (SWaP) of the system and the cost of flight qualification prevent the ALHAT HDS from being used by near-term robotic missions such as Discovery or New Frontiers missions. Fortunately, given their smaller size, robotic landers have simpler HD requirements for imaged area and maximum range that can be satisfied with a compact HDS composed of a simplified HD algorithm, an existing TRL 9 flight processor, and an existing flash LIDAR that will reach TRL 8 in 2016. Efforts are underway within ALHAT to assemble and flight demonstrate a compact HDS in time for the 2016 New Frontiers mission proposal solicitation. The knowledge gained from developing the compact HDS will also feed back into continuing ALHAT work focused on human lander requirements and the development of an HDS for future human and human precursor missions.

The Morpheus flight profile provided the final approach phase of a lunar landing, which involves HDA and precise terminal vertical descent to soft landing at the identified safe site. This phase only tests a small segment of the NDL performance envelope, and does not include the TRN function of ALHAT (for which the LAlt or LVS are intended). Following the flight tests, upgrades to the NDL have been underway to increase the velocity limits from $70 \mathrm{~m} / \mathrm{s}$ to $200 \mathrm{~m} / \mathrm{s}$ and maximum altitude from $2.2 \mathrm{~km}$ to well over $4 \mathrm{~km}$, which meets the needs of a wide range of Mars and lunar landing scenarios. This upgraded NDL will be integrated with LVS and flight tested on a commercial VTB in 2016 through the NASA Flight Opportunities Program. These new flight tests will demonstrate the TRN element of ALHAT, along with the integrated operation of TRN and NDL. The NDL upgrades and the new flight test are part of ALHAT efforts to mature the NDL for infusion into near-term robotic missions.

The infusion of TRN into a spaceflight mission could occur with the adoption of LVS into the Mars 2020 mission. There are also Discovery and New Frontiers missions considering TRN for precision landing 
scenarios. Most of these missions are focused on the passive-optical techniques rather than methods based on the LAlt. At the time of this writing, there are no near-term missions identified that would be interested in the LAlt, although the capability could find application in other areas such as rendezvous and docking.

\section{Closing Remarks}

Closing remarks on the success of the flight test campaign and the forward path to infusion.

The successful completion of the ALHAT flight testing with the Morpheus vehicle has demonstrated the dynamic performance of the HDS, NDL and LAlt. The next steps for each of these sensors will take the technologies closer to spaceflight infusion.

Section will summarize the rest of the paper but not provide any new technical content.

\section{Acknowledgments}

We must acknowledge the extensive efforts of the entire ALHAT and Morpheus teams across NASA and its supporting contractors over the many years of work undertaken to conduct and successfully accomplish the ALHAT flight test objectives. The excellent results discussed within this paper are a tribute to the individual efforts of each and every team member toward the larger goal of developing, implementing and ultimately flight testing all of the ALHAT technologies in dynamically relevant, terrestrial test environments. The work described herein was performed within NASA at the Johnson Space Center, Jet Propulsion Laboratory, Langley Research Center, and Kennedy Space Center as a part of the ALHAT and Morpheus projects. The ALHAT work at the Jet Propulsion Laboratory, California Institute of Technology, was performed under contract with the National Aeronautics and Space Administration (Government sponsorship acknowledged).

\section{References}

\footnotetext{
${ }^{1}$ Committee on the Planetary Science Decadal Survey; Space Studies Board; Division on Engineering and Physical Sciences; National Research Council of the National Academies, Vision and Voyages for Planetary Science in the Decade 2013-2022, The National Academies Press, 2012.

${ }^{2}$ Steering Committee for NASA Technology Roadmaps; National Research Council of the National Academies, NASA Space Technology Roadmaps and Priorities: Restoring NASA's Technological Edge and Paving the Way for a New Era in Space, The National Academies Press, 2012.

${ }^{3}$ Epp, C. D., Robertson, E. A., and Carson III, J. M., "Developing Autonomous Precision Landing and Hazard Avoidance Technology from Concept through Flight-Tested Prototypes," Proc. AIAA GNEC Conference, AIAA 2015-0324, Kissimmee, FL, Jan. 5-8 2015.

${ }^{4}$ Epp, C. and Smith, T., "The Autonomous Precision Landing and Hazard Detection and Avoidance Technology (ALHAT)," Proc. Space Technology and Applications International Forum (STAIF), 2007.

${ }^{5}$ Epp, C., Robertson, E., and Brady, T., "Autonomous Landing and Hazard Avoidance Technology (ALHAT)," Proc. IEEE Aerospace Conference (AEROCONF 2008), March 2008.

${ }^{6}$ Johnson, A., Bergh, C., Cheng, Y., et al., "Design and Ground Test Results for the Lander Vision System," $36^{\text {th }}$ Annual AAS Guidance and Control Conference, AAS 13-042, Breckenridge, CO, Feb. 1-6 2013.

${ }^{7}$ Johnson, A. and Montgomery, J., "An Overview of Terrain Relative Navigation for Precise Lunar Landing," IEEE Aerospace Conference (AEROCONF 2008), March 2008.

${ }^{8}$ Johnson, A. and Ivanov, T., "Analysis and Testing of a LIDAR-Based Approach to Terrain Relative Navigation for Precise Lunar Landing," Proc. AIAA Guidance, Navigation, and Control Conference, August 2011.

${ }^{9}$ Pierrottet, D., Amzajerdian, F., and Barnes, B., "A long distance Laser Altimeter for terrain relative navigation and spacecraft landing," SPIE Defense and Security Symposium, June 2014.

${ }^{10}$ Amzajerdian, F., Petway, D. P. L., Hines, G., and Barnes, B., "Doppler lidar sensor for precision navigation in GPSdeprived environment," Proc. International Society for Optics and Photonics (SPIE), June 2013.

${ }^{11}$ Pierrottet, D. F., Amzajerdian, F., Petway, L. B., Hines, G. D., and Barnes, B., "Field Demonstration of a Precision Navigation Lidar System for Space Vehicles," Proc. AIAA Guidance, Navigation, and Control Conference, Boston, MA, August 2013.

${ }^{12}$ Carson, J. M., Bailey, E. S., Trawny, N., Johnson, A. E., Roback, V. E., Amzajerdian, F., and Werner, R. A., "Operations Concept, Hardware Implementation and Ground-Test Verification of a Hazard Detection System for Autonomous and Safe Precision Lunar Landing," AAS/AIAA Astrodynamics Specialist Conference, AAS 13-856, Hilton Head, SC, Aug. 11-15 2013.

${ }^{13}$ Trawny, N., Carson, J. M., Huertas, A., Luna, M. E., Roback, V. E., Johnson, A. E., Martin, K. E., and Villalpando,
} 
C. Y., "Helicopter Flight Testing of a Real-Time Hazard Detection System for Safe Lunar Landing," Proc. AIAA SPACE 2013 Conference \& Exposition, San Diego, CA, 10-12 Sept. 2013.

${ }^{14}$ Huertas, A., Cheng, Y., and Madison, R., "Passive Imaging Based Multi-cue Hazard Detection for Spacecraft Safe Landing," Proc. IEEE Aerospace Conference, March 2006.

${ }^{15}$ Amzajerdian, F., Pierrottet, D., Petway, L., Hines, G., and Roback, V., "Lidar systems for precision navigation and safe landing on Planetary Bodies," Proc. International Society for Optics and Photonics (SPIE), August 2011.

${ }^{16}$ Keim, J., Mobasser, S., Kuang, D., Cheng, Y., Ivanov, T., Johnson, A., Goldberg, H., Khanoyen, G., and Natzic, D., "Field test implementation to evaluate a flash LIDAR as a primary sensor for safe lunar landing," IEEE Aerospace Conference (AEROCONF 2010), March 2010.

${ }^{17}$ Ivanov, T., Huertas, A., and Carson, J., "Probabilistic Hazard Detection for Autonomous Safe Landing," Proc. AIAA Guidance, Navigation, and Control Conference, August 2013.

${ }^{18}$ Stettner, R., Bailey, H., and Richmond, R., "Eye Safe Laser Radar Focal Plane Array for Three-Dimensional Imaging," Proc. International Society for Optics and Photonics (SPIE), 2001.

${ }^{19}$ Rutishauser, D. K., Epp, C. D., and Robertson, E. A., "Free-Flight Terrestrial Rocket Lander Demonstration for NASA's Autonomous Landing and Hazard Avoidance Technology (ALHAT) System," Proc. AIAA SPACE 2012 Conference 83 Exposition, September 2012.

${ }^{20}$ Roback, V. E., Bulyshev, A. E., Amzajerdian, F., Brewster, P. F., Barnes, B. W., Kempton, K. S., and Reisse, R. A., "Helicopter Flight Test of a Compact, Real-Time 3-D Flash Lidar for Imaging Hazardous Terrain during Planetary Landing," Proc. AIAA SPACE 2013 Conference \& Exposition, San Diego, CA, 10-12 Sept. 2013.

${ }^{21}$ Roback, V. E., Bulyshev, A. E., Amzajerdian, F., and Reisse, R. A., "Helicopter Flight Test of 3D Imaging Flash Lidar Technology for Safe, Autonomous, and Precise Planetary Landing," Proc. SPIE 8731, Laser Radar Technology and Applications XVIII, Vol. 87310H, doi:10.1117/12.2015961, June 4 2013, pp. 1-20.

${ }^{22}$ Pierrottet, D., Amzajerdian, F., Petway, L., Barnes, B., and Lockard, G., "Flight test performance of a high precision navigation Doppler lidar," Proc. SPIE 7323, Laser Radar Technology and Applications XIV, Vol. 732311, doi:10.1117/12.821902, May 62009.

${ }^{23}$ Carson III, J. M., Robertson, E. A., Pierrottet, D. F., Roback, V. E., Trawny, N., Devolites, J. L., Hart, J. J., Estes, J. N., and Gaddis, G. S., "Preparation and Integration of ALHAT Precision Landing Technology for Morpheus Flight Testing," Proc. AIAA SPACE 2014 Conference \& Exposition, San Diego, CA, Aug. 5-7 2014.

${ }^{24}$ Carson III, J. M., Hirsh, R. L., Roback, V. E., Villalpando, C. Y., Busa, J. L., Pierrottet, D. F., Trawny, N., Martin, K. E., and Hines, G. D., "Interfacing and Verifying ALHAT Safe Precision Landing Systems with the Morpheus Vehicle," Proc. AIAA GNEC Conference, Kissimmee, FL, Jan. 5-8 2015.

${ }^{25}$ Luna, M. E., Huertas, A., Trawny, N., Villalpando, C. Y., Martin, K. E., Wilson, W., and Restrepo, C. I., "Simulations of the Hazard Detection System for Approach Trajectories of the Morpheus Lunar Lander," Proc. AIAA GNEC Conference, AIAA 2015-0326, Kissimmee, FL, jan 5-8.

${ }^{26}$ Trawny, N., Huertas, A., Luna, M. E., Villalpando, C. Y., Martin, K., Carson, J. M., Johnson, A. E., Restrepo, C., and Roback, V. E., "Flight testing a Real-Time Hazard Detection System for Safe Lunar Landing on the Rocket-Powered Morpheus Vehicle," Proc. AIAA GNEC Conference, AIAA 2015-0326, Kissimmee, FL, jan 5-8. 